\title{
Management of traumatic double-level spondyloptosis of the thoracic spine with posterior spondylectomy: case report
}

\begin{abstract}
Abolfazl Rahimizadeh, MD, ${ }^{1}$ and Ava Rahimizadeh, BS ${ }^{2}$
${ }^{1}$ Department of Neurosurgery, Pars Advanced \& Minimally Invasive Manners Research Center, Pars Hospital, Iran University of Medical Sciences, Tehran, Iran, and 2University of Birmingham, United Kingdom

Traumatic spondyloptosis of the thoracic spine is an uncommon injury. In most cases, spondyloptosis is confined to one vertebral body, whereas double-level spondyloptosis is extremely rare. Most patients who sustain traumatic spondyloptosis immediately become paraplegic, but in some cases neurological function is preserved. If this occurs, it is due to detachment of the pedicles from the corresponding vertebral body, resulting in spontaneous decompression of neural elements.

Herein, the authors describe a case of undetected traumatic double-level spondyloptosis in the upper thoracic region in an adult male patient who was neurologically intact for 2 days but later became paraplegic. Initially, management of this pathology seemed a very challenging scenario. However, with review of the reconstructed CT images and reproduction of the injury on a plastic model, a posterior-only approach was chosen as an alternative operative solution for this catastrophic injury. Via this single-stage posterior approach, long-segment pedicle screw/rod instrumentation resulted in successful reduction, restoration of alignment, and stabilization after 1-level posterior spondylectomy.

To the best of the authors' knowledge, this is the first example reported in the literature of double-level spondyloptosis of the thoracic and the lumbar spine. This report describes the rationale, mechanism, and technical details afforded for reduction and stabilization of this rare injury.
\end{abstract}

http://thejns.org/doi/abs/10.3171/2015.3.SPINE14183

KEY WORDS spinal trauma; spine shortening; spondylectomy; spondyloptosis; thoracic fracture; vertebral column resection; vertebrectomy

$\mathrm{T}$ RAUMATIC spondyloptosis is defined as complete dislocation of one vertebral body anterior, posterior, or lateral to the caudal vertebra. ${ }^{1,4,9,10,13,16,18,21,22}$ It is caused by significant high-energy trauma and is a rare condition, particularly in the thoracic spine., $10,13,16,18$ The thoracic spine is a very unusual region for the occurrence of this type of injury because of its rigid configuration, which is mainly attributable to the ribs and thick ligaments and the sagittal orientation of the facet joints., ${ }^{9,10,13,16,18}$ Therefore, traumatic telescopic descent of 2 vertebral bodies, termed double spondyloptosis, is an extremely rare event.

Unstable spinal fractures and, in particular, those that involve spondyloptosis, are frequently associated with complete cord injury. This is particularly true in the thoracic spine, where there is the least space around the neural elements..$^{6,11-16}$ In rare cases, however, a patient sustaining such a severe spinal injury remains neurologically normal. $5,8-10,12-16,18$ The mechanism of preservation of normal neurological status involves free-floating vertebral arches,

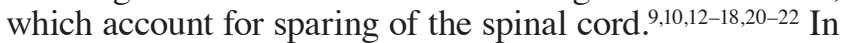
such circumstances bilateral pedicular shear, designated as saving fractures of the vertebral arch, allows the posterior elements to remain almost aligned, which may preserve spinal canal integrity and normal neurological function despite gross displacement of the corresponding vertebral body. ${ }^{5,12-18}$ 
Herein the authors describe their experience with a 29-year-old man with traumatic upper thoracic double posterior spondyloptosis, who was neurologically normal in the first 2 days after trauma but became paraplegic once he attempted to walk. Initially, reduction and restoration of the anatomical alignment seemed a very challenging scenario in this injury, which became more severe because of delayed diagnosis in this specific case. However, with reproduction of the injury on a plastic model it was found that posterior spinal shortening via 1-level spondylectomy was a proper strategy for the management of this catastrophic injury. With the use of this method, the injury could be reduced, aligned, corrected, and fused after 1-level posterior spondylectomy. To the best of our knowledge, neither double spondyloptosis nor its successful reduction after 1-level posterior spondylectomy has been reported previously.

\section{Case Report}

Presentation and Examination

A 29-year-old man sustained moderate head trauma after falling from a 2-story building. The patient became unconscious immediately thereafter and was transferred to an intensive care unit of the nearest hospital, where his brain CT scan showed brain edema. He gradually regained consciousness within 48 hours. At this time, examination showed that his neurological status was intact, but he complained of mild low-neck pain. To address this complaint, a whole-spine radiograph was obtained and interpreted to be normal. Therefore, he was transferred to the regular hospital floor on the 3rd postadmission day, where he was persuaded to walk. Unfortunately, within a minute or two after standing, he felt ascending numbness in his lower extremities and became paraplegic, with a complete sensory level above the nipples. At the request of the patient's physician and relatives, he was admitted to our hospital. On admission his neurological status, assessed using the scoring system of the American Spinal Injury Association
(ASIA), was Grade A, with complete motor and sensory deficits below the T-2 level.

According to the clinical picture, an injury affecting the upper thoracic region was suspected, and CT and reconstructed CT combined with CT angiography of the cervicothoracic region were performed. Axial CT images showed the characteristic "double-sun" or double-vertebra appearance of spondyloptosis (Fig. 1C). Reconstructed sagittal CT images showed bilateral fractures at the pedicle-vertebral body junction at T-2, T-3, and T-4; also noted were detachment and subsequent free descent of the corresponding intact vertebral bodies of T-1 and T-2 into the posterior aspect of the T-3 and T-4 vertebral bodies (Fig. $1 \mathrm{~A}$ and $\mathrm{B})$.

\section{Injury Reconstruction Using a Model}

For better understanding of the events and to clarify how neurological function could have remained initially intact, reconstruction of the injury on a plastic model was done. With this virtual reconstruction we determined that avulsion and detachment of the posterior elements from the vertebral bodies might result in free-floating arches, which may have preserved the integrity of the spinal cord despite spondyloptosis (Fig. 2). This type of injury was postulated to have resulted from an anteroposterior shear that occurred through the T2-3 intervertebral disc. Concomitant hyperextension may have caused bilateral fractures at the pedicle-body junction at 3 levels or free-floating arches with preservation of the cord function despite subsequent vertebral body ptosis at 2 levels.

Surgical reduction of this spinal injury was anticipated to be impossible because of the severity of spondyloptosis and the distance between the cranial and caudal vertebral bodies. However, different approaches and techniques were tried on a model, where it was determined that 1-level spondylectomy via a posterior-only approach seemed to be the best solution. After this approach was chosen, posterior spondylectomy of T-2, reduction and apposition of the cranial (T-1) and caudal (T-3) vertebral bodies, and
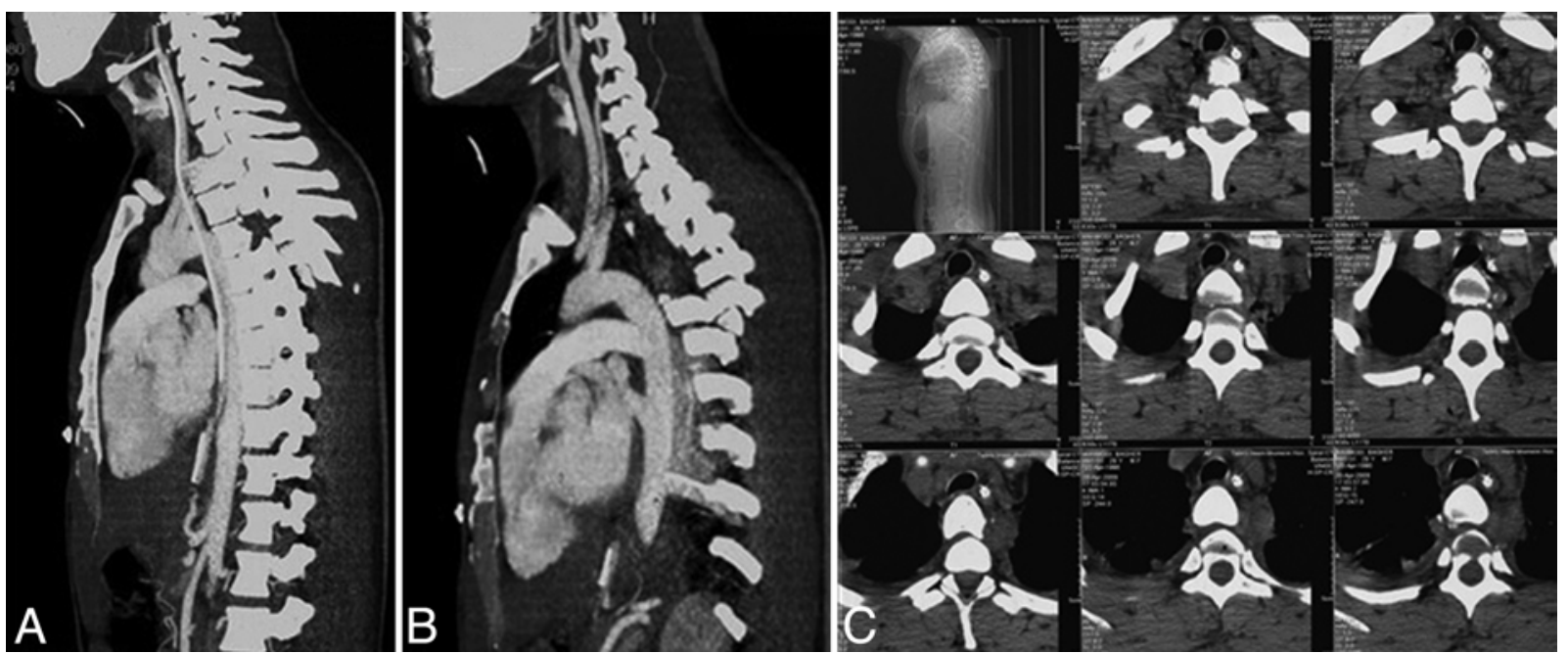

FIG. 1. Sagittal CT reconstructions and axial CT scans of the cervicothoracic region. A: Free descent of the T-1 and T-2 vertebral bodies located posterior to the T-3 and T-4 vertebral bodies. B: Detachment of the T-1, T-2, and T-3 pedicles from their corresponding bodies. C: Double-sun appearance that is a characteristic feature of spondyloptosis. 


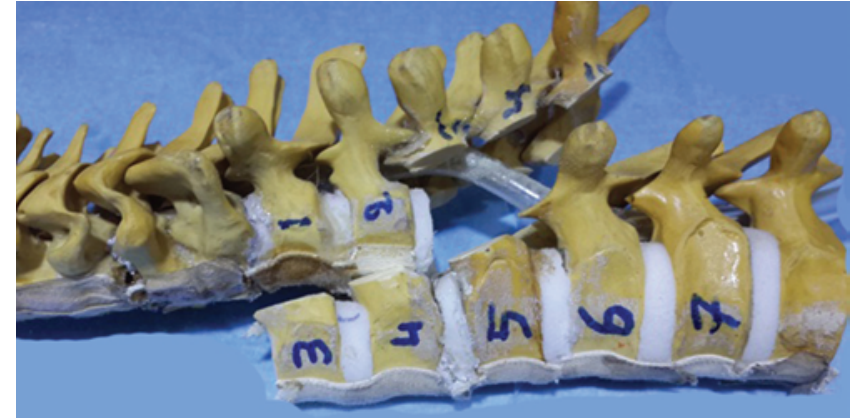

FIG. 2. Reconstruction of the lesion on a model shows how spontaneous decompression of the spinal cord might occur. Note that the model indicates that continuity of the cord could be preserved. Figure is available in color online only.

long-segment screw/rod stabilization were performed successfully on the model. Packing of the intervertebral gap between T- 1 and T-3 with a local autogenous bone graft was determined to be an effective way to achieve the anterior column reconstruction (Fig. 3).

\section{Operation and Technique}

After receiving general anesthesia, the patient was po- sitioned prone. After stripping of the muscles from C-6 to $\mathrm{T}-7$, the loose and free-floating posterior elements of T-3, T-4, and T-5 were excised en bloc with the 1-piece removal of the posterior arch of T-2. Surprisingly, the spinal cord was visualized with an intact dural covering and even without lacerations, findings that indicated spontaneous decompression of the cord, which can be seen in this type of injury.

Subsequently, lateral mass screws at C-6 and pedicle screws at C-7 and T-1 were placed bilaterally. Then, thoracic pedicle screws were inserted from T- 5 to $\mathrm{T}-7$ on both sides and a temporary rod was assembled on one side, extending from C-6 to T-7.

Later, en bloc removal of the T-2 vertebral body was done. A temporary rod on the contralateral side was assembled, and with the application of gentle distraction through T-1 to T-3 the corresponding vertebral bodies were approximated and also opposed each other with proper alignment of the vertebral column. Then, after removal of one of the temporary rods, T-3 and T-4 screws could be inserted on the contralateral side. The same procedure was repeated on the other side, with the final construct extending from C-6 to T-7 with permanent rods. At this stage, local autogenous bone grafts shaped appropriately were packed into the T1-3 intervertebral gap to secure the ante-
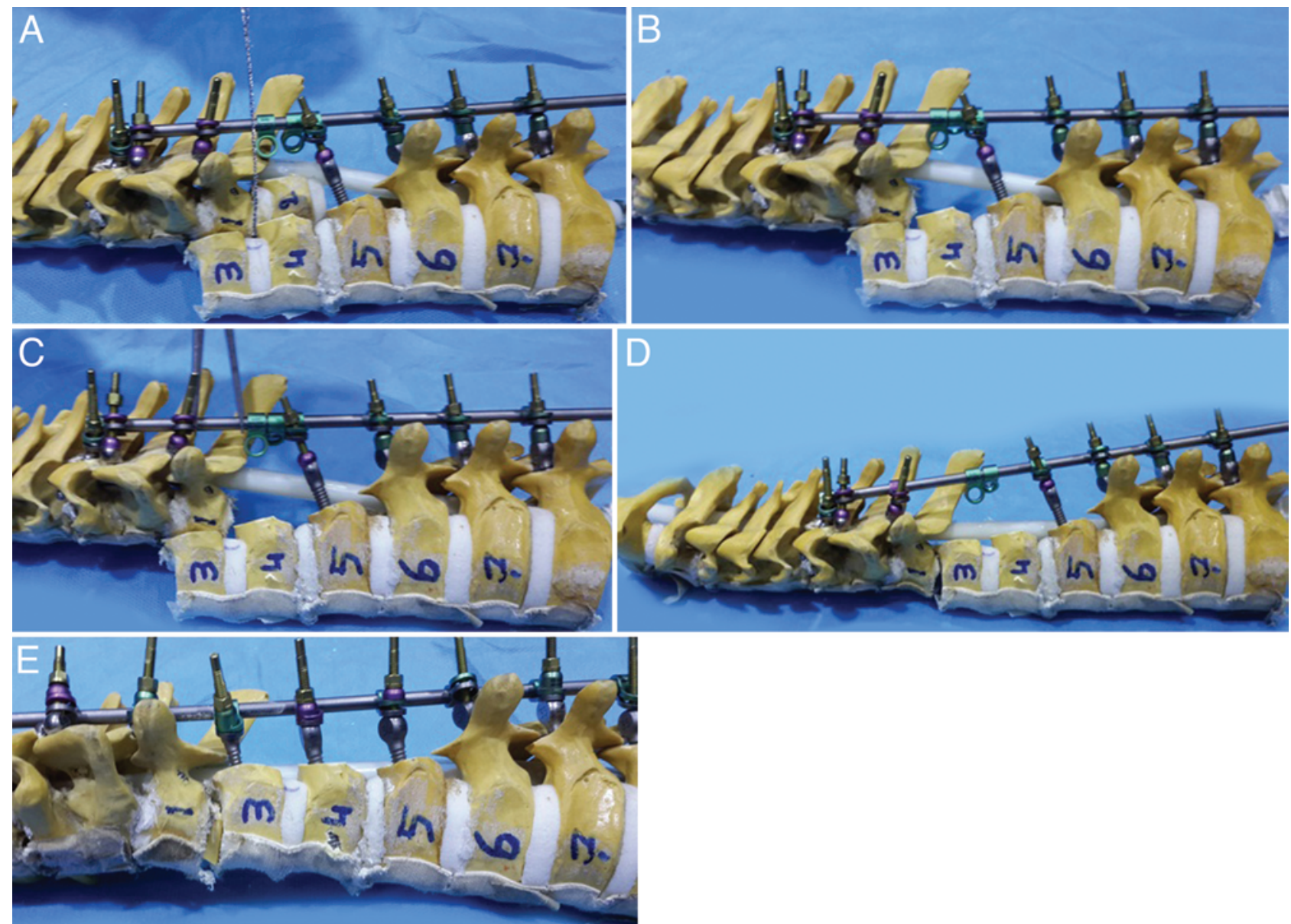

FIG. 3. Steps of surgery planned preoperatively on a model. After the performance of T-2 vertebral body spondylectomy (A and $\mathrm{B})$, distraction is applied (C), and then the injury is easily reduced with approximation of T-1 to T-3 (D). A local bone graft is packed in the gap between T-1 and T-3 (E). Figure is available in color online only. 
rior vertebral column reconstruction, and subsequently the construct was finalized in compression. Two cross-links connecting the rods were used later. Postoperative plain radiography demonstrated proper vertebral alignment and instrumentation placement (Fig. 4).

\section{Postoperative Course}

The patient's postoperative clinical course was uneventful. A few days after surgery, sitting exercises were initiated using an extended Minerva orthosis. Later, he was transferred to a rehabilitation center.

Reconstructed CT images obtained at the 2-year follow-up showed good alignment, fusion, and stabilization, with evidence of graft incorporation in the T1-3 intervertebral space (Figs. 5 and 6).

The patient's clinical status was still ASIA Grade A, with complete paraplegia below $\mathrm{T}-2$, but he was coping well with the loss of lower-extremity function and had returned to his previous job in a wheelchair.

\section{Discussion}

Traumatic spondyloptosis is defined as complete subluxation of one vertebral body with respect to an adjacent vertebral body either in the coronal or the sagittal plane . $^{1,9,10,13,16,18,21,22}$ In sagittal-plane spondyloptosis, the upper vertebral body is usually located anterior to the caudal vertebra, whereas lateral and posterior spondyloptosis have been sparsely reported. ${ }^{1,3,4,9,10,13,16,18,20-22}$ Thoracic spine spondyloptosis described in the literature has occurred most frequently in the sagittal plane and in an anterior direction and less frequently in the coronal plane and rarely in the posterior direction. According to Denis and Burkus, the shear injuries are subdivided into posteroanterior and anteroposterior subtypes depending on the direction of the impact. ' According to these authors, the less
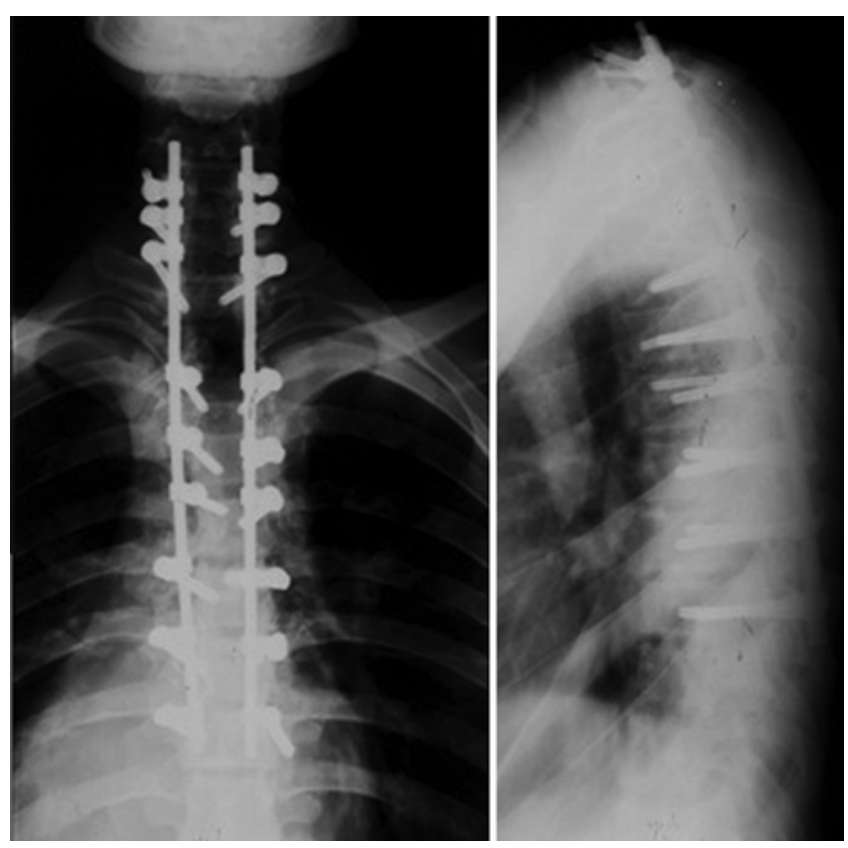

FIG. 4. Postoperative cervicothoracic plain radiographs, anteroposterior (left) and lateral (right) views. common anteroposterior shear is usually associated with forceful hyperextension with multiple fractures of the posterior column and free-floating arches.

The thoracic spine has significant inherent stability provided by the anterior and posterior longitudinal ligaments, rib cage, and thick ligamentum flavum and the orientation of the facet joints. ${ }^{8-10,12,16,18}$ Therefore, in this region, substantial force is required to cause spondyloptosis and considerable violence to produce double spondyloptosis. Motor vehicle accidents and substantial falls, with their capability to induce considerable shear forces, are the most frequent causes of traumatic spondyloptosis., ${ }^{9}, 10,13,16,18$

Biomechanically, the mechanism of double-level spondyloptosis in this particular patient was most likely to have been a severe shear force in the anteroposterior direction combined with hyperextension, with complete discoligamentous disruption occurring at the T2-3 intervertebral disc, resulting in complete posterior dislocation of the T-1 and T-2 vertebrae with respect to the T-3 and T-4 vertebrae. Eventually, descent of the cranial vertebrae had cut off the pedicles of the lower vertebrae from their bodies, resulting in free-floating posterior arches, hence permitting the free descent and relocation of the T-1 and T-2 vertebral bodies in the posterior aspect of the T-3 and T-4 vertebral bodies.

The narrower canal in the thoracic spine and tenuous blood supply to the spinal cord at this region allows high-energy trauma to induce complete neurological injury and irreversible paraplegia in a majority of the victims. ${ }^{2,5,6,8-10,12-18}$

Under certain rare circumstances, however, despite significant anterior-posterior or lateral displacement, the patient can remain neurologically intact or be only mildly affected.9,10,12,14,17,18 The reason for such neural sparing is quite interesting because a great discrepancy exists between the spectacular radiological features and the neurological picture.

In 1948, Bohler was the first to describe the possible mechanism that plays the major role in preservation of the integrity of spinal cord function despite gross fracture dislocation of the spine, and he designated this as saving fractures of the neural arches. ${ }^{12}$

Later, in 1976, Sir Ludwig Guttman reported 2 more cases in which, despite gross lateral displacement, one patient was neurologically intact and the other showed only a mild neural deficit, demonstrating that the neural arch fracture was the cardinal factor in neurological sparing. ${ }^{9,14}$ Since the publication of Guttman's cases, only a few cases of traumatic thoracic spondyloptosis in patients with normal neurological status have been reported., ${ }^{9} 10,12,14,17,18$ According to these reports, bilateral fractures at the vertebral body-pedicle junction exert a protective effect by enlarging the canal through spontaneous decompression of the spinal cord. Through these consecutive events, the posterior elements maintain their continuity, sparing the integrity of the spinal cord and preserving neurological function despite gross displacement of the vertebral body.

In such instances, clinical examinations often fail to reveal any notch or gibbus, particularly when the spinal processes and paraspinal muscles remain in alignment. Even local tenderness may be absent. Therefore, missing such a 

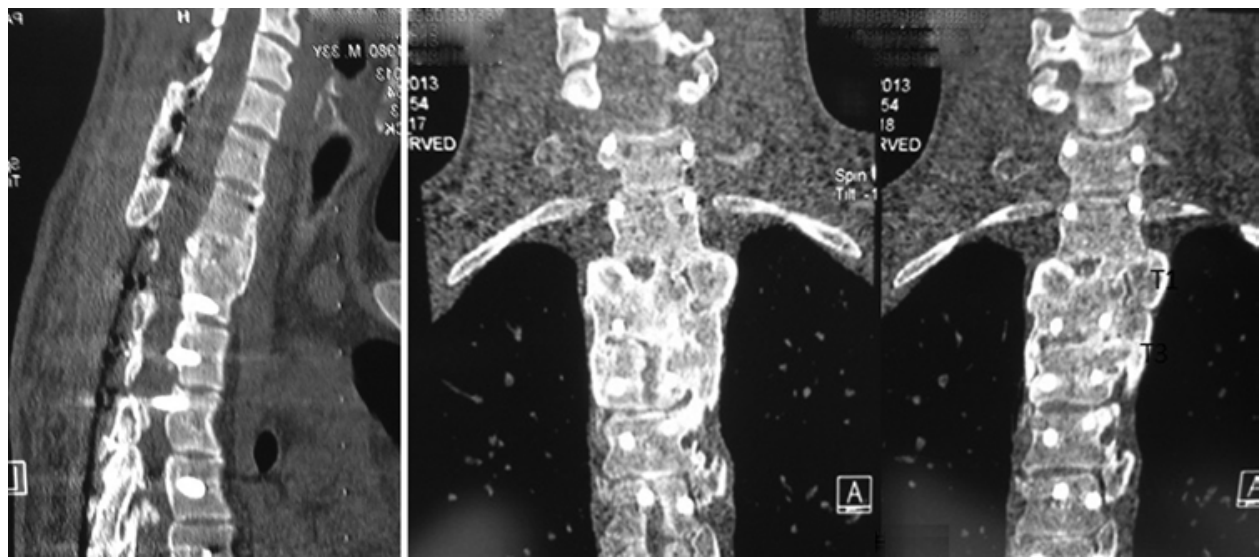

FIG. 5. 2D reconstructed CT images at 2-year follow-up. Sagittal (left) and coronal (right) views both show proper alignment and fusion. $A=$ anterior

highly unstable spinal injury is always possible. . $^{2,10,12,14,15}$ The presence of concomitant injuries such as rib fractures, pneumothorax, and hemothorax may also cause spinal injuries to be overlooked.

Radiologically, major spinal injuries in the upper thoracic region are often difficult to diagnose and might be missed initially because of the musculature of the region and the shadow of the scapula overlapping with the upper thoracic spine. . $^{2,8,15}$

In spondyloptosis, axial CT views can clearly demonstrate the classic double-vertebra sign or double-sun appearance, delineating fractures of the pedicles and fractures of the posterior arch. Reconstructed CT images give precise and comprehensive delineation of the pedicle fractures, displaced vertebral body, and degree of spinal cord compromise. CT angiography may be necessary to exclude associated aortic injury. MRI in spondyloptosis can show the associated discoligamentous injury and cord myelomalacia.

The primary goals in the management of spondyloptosis are reduction, alignment, and stabilization. ${ }^{2,5,6,8,13,15}$ However, surgery in patients with intact neural function
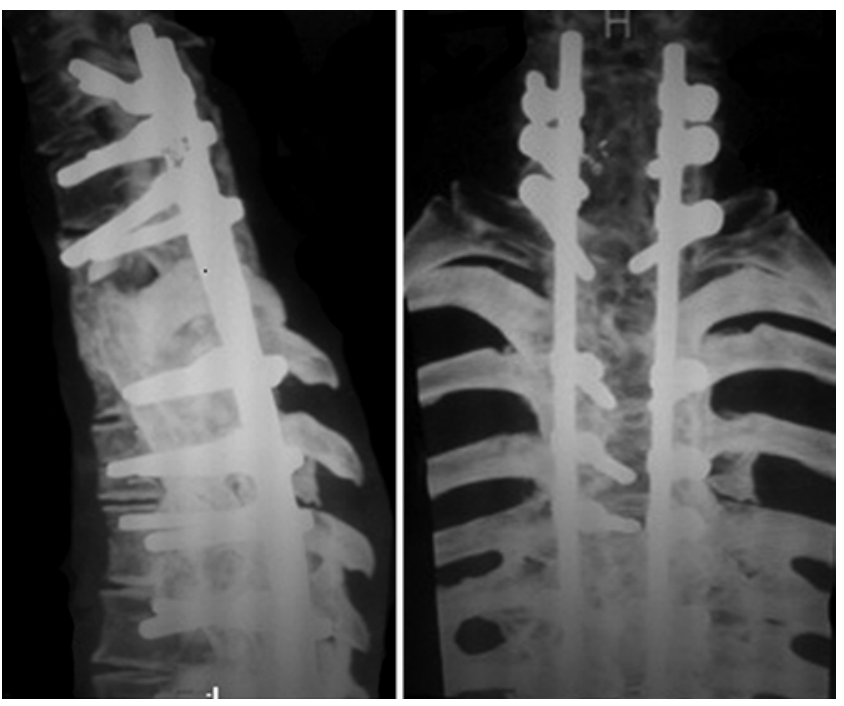

FIG. 6. 3D reconstructed CT images. Sagittal (left) and coronal (right) images demonstrate the surgical construct used in this patient. is an extremely challenging process, particularly in those with normal neurological function, in whom preservation of neurological integrity is paramount. , $, 9,10,12,14,16-18,20-22$ In such instances, surgical intervention should be carried out under neuromonitoring to avoid further injury to the spinal cord. However, in paraplegic patients with spondyloptosis, the foremost goal is stabilization after reduction and alignment, surgical interventions that allow early mobilization and rehabilitation with a wheelchair.

For a better understanding of their management, cases of spondyloptosis can be be classified as reducible or irreducible. In reducible cases, because of associated severe ligamentous disruption, reduction can be easily achieved with open distraction applied through a posterior approach. ${ }^{2,4,21,22}$ In irreducible cases, distraction may be ineffective when reduction of the affected vertebrae is made more difficult by bony fractures associated with the injury or by severe adhesions caused by scar tissue seen in patients with chronic spondyloptosis for whom there has been a considerable delay in diagnosis or surgical management.

In reducible spondyloptosis, the distraction between the segmental pedicle screws may provide sufficient reduction force to allow proper restoration of anatomical alignment of the dislocated vertebral body. 2,4,21,22 Interbody and posterolateral fusion also facilitates arthrodesis. However, considering the high rate of instability, the use of extended instrumentation encompassing a minimum of 3 vertebrae below and above the affected vertebra is advised.

Whenever the inefficacy of distraction applied to a posterior construct is confirmed, the case can be designated as irreducible. In such instances, same-stage corpectomy of the dropped vertebral body is advised. After corpectomy, restoration of alignment can be easily achieved with approximation of the vertebral bodies located cranial and caudal to the corpectomized body and subsequent spinal shortening, ${ }^{2,4,15}$ with the addition of an appropriately shaped autogenous local bone graft placed into the intervertebral space to facilitate fusion. In cases with a large gap between the vertebral bodies, placement of an expandable cage is advised. ${ }^{20}$ In the past, the anterolateral approach has been the most acceptable corridor affording access for the achievement of corpectomy. ${ }^{20}$ However, 
posterior spondylectomy seems a safe and useful alternative, which appears to obviate the need for staged surgery in irreducible cases. ${ }^{3,19}$

Preoperative application of pelvic traction and its continuation during surgery to facilitate reduction have been advised in patients with irreducible injury. Preoperative traction may ameliorate spinal column shortening and prevent soft-tissue contraction around the lesion and facilitate its open reduction. ${ }^{11}$

A review of the literature suggests that significant recovery is not unusual in patients with thoracic spondyloptosis who have suffered an incomplete spinal cord injury. Obviously, preoperative function is a fairly good predictor of neurological outcome., ${ }^{4,615}$ On the other hand, the clinical outcome with regard to neurological recovery seems unfavorable in paraplegic patients with thoracic spondyloptosis, such as the case reported here., ${ }^{2,6,15}$ With good reduction and proper stabilization, however, this patient was able to continue his work and his social interactions in a wheelchair.

In summary, traumatic double-level spondyloptosis of the thoracic spine should be regarded as an extremely rare event, and to our knowledge it has not been previously reported. Even with such a spectacular injury, bilateral pedicle fractures and free-floating arches may allow the spinal cord to escape damage leading to neurological deficits.

Furthermore, we believe that review of reconstructed CT images and reproduction of the injury on a plastic model can play important roles in enabling understanding of unusual spinal injuries and facilitating proper surgical decision making. Finally, posterior 1-level spondylectomy should be considered a useful alternative in management of traumatic irreducible single- or double-level spondyloptosis of the thoracic or lumbar spine.

\section{References}

1. Ahmed A, Mahesh BH, Shamshery PK, Jayaswal A: Traumatic retrolisthesis of the L4 vertebra. J Trauma 58:393394, 2005

2. Alobaid A, Arlet V, Ouellet J, Reindl R: Surgical technique. Technical notes on reduction of thoracic spine fracture dislocation. Can J Surg 49:131-134, 2006

3. Barberá J: T12-L1 telescoped chronic dislocation treated by en bloc one-piece spondylectomy and spine shortening. J Spinal Disord Tech 17:163-166, 2004

4. Bellew MP, Bartholomew BJ: Dramatic neurological recovery with delayed correction of traumatic lumbar spondyloptosis. Case report and review of the literature. J Neurosurg Spine 6:606-610, 2007

5. Bohlman HH: Treatment of fractures and dislocations of the thoracic and lumbar spine. J Bone Joint Surg Am 67:165169,1985

6. Bohlman HH, Freehafer A, Dejak J: The results of treatment of acute injuries of the upper thoracic spine with paralysis. J Bone Joint Surg Am 67:360-369, 1985

7. Denis F, Burkus JK: Shear fracture-dislocations of the thoracic and lumbar spine associated with forceful hyperextension (lumberjack paraplegia). Spine (Phila Pa 1976) 17:156-161, 1992
8. el-Khoury GY, Whitten CG: Trauma to the upper thoracic spine: anatomy, biomechanics, and unique imaging features. AJR Am J Roentgenol 160:95-102, 1993

9. El Masri WS, Silver JR: Traumatic spondyloptosis of the dorsal spine with incomplete neurological deficit. Injury 15:35-37, 1983

10. Gitelman A, Most MJ, Stephen M: Traumatic thoracic spondyloptosis without neurologic deficit, and treatment with in situ fusion. Am J Orthop 38:E162-E165, 2009

11. Hutchinson MR, Dall BE: Fracture-dislocation of the thoracic and lumbar spine: advantages of halo-bifemoral traction. J Spinal Disord 6:482-488, 1993

12. Korovessis P, Sidiropoulos P, Dimas A: Complete fracturedislocation of the thoracic spine without neurologic deficit: case report. J Trauma 36:122-124, 1994

13. Lee CW, Hwang SC, Im SB, Kim BT, Shin WH: Traumatic thoracic spondyloptosis: a case report. J Korean Neurosurg Soc 35:622-624, 2004

14. London PS: Fracture-dislocations of the spine that may spare the cord: an explanation. Injury 24:347-349, 1993

15. Sapkas GS, Papagelopoulos PJ, Papadakis SA, Themistocleous GS, Stathakopoulos DP, Efstathiou P, et al: Thoracic spinal injuries: operative treatments and neurologic outcomes. Am J Orthop (Belle Mead NJ) 32:85-88, 2003

16. Sekhon LH, Sears W, Lynch JJ: Surgical management of traumatic thoracic spondyloptosis: review of 2 cases. J Clin Neurosci 14:770-775, 2007

17. Shapiro S, Abel T, Rodgers RB: Traumatic thoracic spinal fracture dislocation with minimal or no cord injury. Report of four cases and review of the literature. J Neurosurg 96 (3 Suppl):333-337, 2002

18. Simpson AH, Williamson DM, Golding SJ, Houghton GR: Thoracic spine translocation without cord injury. J Bone Joint Surg Br 72:80-83, 1990

19. Wang F, Zhu Y: Treatment of complete fracture-dislocation of thoracolumbar spine. J Spinal Disord Tech 26:421-426, 2013

20. Wilkinson JS, Riesberry MA, Mann SA, Fourney DR: Traumatic lateral expulsion of the L-4 vertebral body from the spinal column. J Neurosurg Spine 14:508-512, 2011

21. Yadla S, Lebude B, Tender GC, Sharan AD, Harrop JS, Hilibrand AS, et al: Traumatic spondyloptosis of the thoracolumbar spine. J Neurosurg Spine 9:145-151, 2008

22. Zhou TH, Tang X, Xu YQ, Zhu YL: Traumatic spondyloptosis of L4. Spine (Phila Pa 1976) 35:E855-E859, 2010

\section{Disclosure}

The authors report no conflict of interest concerning the materials or methods used in this study or the findings specified in this paper.

\section{Author Contributions}

Conception and design: Abolfazl Rahimizadeh. Analysis and interpretation of data: both authors. Drafting the article: Ava Rahimizadeh. Critically revising the article: Ava Rahimizadeh. Administrative/technical/material support: Abolfazl Rahimizadeh. Study supervision: Abolfazl Rahimizadeh.

\section{Correspondence}

Abolfazl Rahimizadeh, 67 Keshwarz Blvd., Tehran 1415944911, Iran.email: a_rahimizadeh@hotmail.com. 